\title{
Caspase-8 expression is predictive of tumour response to death receptor 5 agonist antibody in Ewing's sarcoma
}

\author{
Zhigang Kang ${ }^{1,2}$, Seth D Goldstein ${ }^{3}$, Yunkai Yu ${ }^{1}$, Paul S Meltzer ${ }^{1}$, David M Loeb ${ }^{3}$ and Liang Cao*,1 \\ ${ }^{1}$ Genetics Branch, Center for Cancer Research, National Cancer Institute, Bethesda, MD 20892, USA; ${ }^{2}$ Basic Science Program, \\ Leidos Biomedical Research, Inc, Frederick, MD 21702, USA and ${ }^{3}$ Department of Oncology, Sidney Kimmel Comprehensive \\ Cancer Center, Johns Hopkins University, Baltimore, MD 21231, USA
}

Background: Despite good initial response to chemotherapy, 30\% of Ewing's sarcoma (EWS) patients with localised tumours develop recurrent disease, associated with poor prognosis. The aim of this study was to address this challenge by conducting preclinical evaluation of a death receptor targeted agent in vitro and in vivo, and by identifying predictive biomarkers.

Methods: Cell viability assays, drug dose responses, immunoblots, rescue with gene transfer, mice tumour models, and statistical comparisons of tumour growth and Kaplan-Meier survival curves.

Results: This study shows that many EWS cell lines are selectively sensitive to a death receptor DR5 antibody and are more resistant to a DR4 antibody. Preclinical evaluation of these cell lines indicates their sensitivity to human DR5 agonist antibody conatumumab in vitro, which induces rapid activation of caspase- 8 and apoptosis. We also found that sensitivity to conatumumab correlates with expression of caspase-8. Furthermore, the catalytic activity of caspase-8 is both necessary and sufficient to confer this sensitivity. In vivo, conatumumab is active against an EWS cell line and a patient-derived xenograft with higher caspase-8 expression, but is not effective against another with lower caspase-8 expression.

Conclusions: These studies suggest the potential of conatumumab as a therapeutic agent against EWS and caspase-8 as a predictive biomarker for sensitivity.

Apoptosis is important in the regulation of various physiological and pathological conditions. The extrinsic apoptotic pathway involves cell-surface receptors, including tumour necrosis factor (TNF) receptor, TNF-related apoptosis-inducing ligand (TRAIL) receptors, and CD95 (Fas) (Locksley et al, 2001). In the case of TRAIL receptors, the binding of the TRAIL ligand to death receptor DR4 or DR5 results in the recruitment of the death-induced signaling complex (DISC), which includes the adaptor protein FADD and initiator proteins caspase- 8 or -10 (Kischkel et al, 2000, 2001). Due to the potential selectivity of TRAIL for cancer cells, there has been a significant interest in agents targeting TRAIL receptors for cancers (Ashkenazi, 2008; Ashkenazi and Herbst, 2008). Some studies suggest that sensitivity to TRAIL could be controlled mainly by upstream events including DISC assembly and caspase-8 activation (Hughes et al, 2009). In addition, multiple factors have been suggested to affect TRAIL-induced apoptosis, including post-translational modifications of DR4 and DR5 receptors, such as O-glycosylation (Wagner et al, 2007) and endocytosis (Zhang and Zhang, 2008), the ubiquitination of initiator caspase-8 (Jin et al, 2009), and c-FLIP that may compete for the recruitment of caspase- 8 and caspase-10 at the DISC (Safa et al, 2008). Further studies are needed to facilitate the identification and implementation of predictive biomarkers for the preclinical and clinical development of TRAIL receptor targeted agents for cancer. 
Ewing's sarcoma (EWS) is the second most common malignant bone tumour in children and young adults. It appears to arise from primitive mesenchymal precursor cells (Tirode et al, 2007), having a common pathogenic mechanism that is nearly always associated with translocations between EWSR1 and ETS family genes, most frequently involving EWSR1 and FLI1 (Delattre et al, 1992). EWS responds well initially to cytotoxic chemotherapy, however, about $30 \%$ of patients with localised tumours develop recurrences, and $65-80 \%$ of patients with metastatic tumours die within 5 years of diagnosis (Balamuth and Womer, 2010). In spite of intensive chemotherapies, the survival rates for relapsed or metastatic EWS have not changed over the past 30 years (Cotterill et al, 2000); thus, targeted therapies are needed. Several studies have explored the feasibility of targeting TRAIL receptors in EWS and to uncover biomarkers predictive of response. An earlier investigation indicated that nearly $80 \%$ of a large panel of EWS cell lines was sensitive to TRAIL-induced apoptosis in vitro (Van Valen et al, 2000). A subsequent study showed that there is variable CASP8 expression in EWS cell lines, and re-expression of CASP8 via gene transfection or demethylation leads to increased sensitivity to TRAIL (Fulda et al, 2001). More recently, cationic lipid-mediated TRAIL gene transfer was shown to prevent EWS xenograft development and increases mouse survival (Picarda et al, 2010). Various agonist therapeutic antibodies against death receptors exhibit anti-tumour activities in preclinical models (Adams et al, 2008; Jin et al, 2008; Luster et al, 2009; Kaplan-Lefko et al, 2010) and are in clinical development (Ashkenazi and Herbst, 2008). Studies on the identification of a specific death receptor that mediates TRAIL-induced cell death in EWS have not been conclusive (Mitsiades et al, 2001; Picarda et al, 2012), and direct evidence supporting the targeting of specific death receptors with antibodies is lacking. Furthermore, preclinical evaluation of a therapeutic compound that would include in vitro studies, predictive biomarker analysis, and in vivo evaluation is needed. Conatumumab is a fully human agonist antibody to death receptor DR5 that has been shown to induce apoptosis in multiple tumour types in vitro and to inhibit xenograft growth in mice (Kaplan-Lefko et al, 2010). Clinical trials with conatumumab showed that it is well tolerated as single agent (Herbst et al, 2010b) or in combination therapy (Cohn et al, 2013; Paz-Ares et al, 2013), yet, a clear demonstration of clinical activity is still lacking. To further explore the potential of conatumumab for the treatment of cancer, we performed a preclinical investigation on EWS. The results illustrate that EWS may be a rational target for conatumumab and other DR5 targeted agents. Our data further suggest that caspase- 8 may be a predictive marker of sensitivity to conatumumab and DR5-targeted agents, because the expression and activity of caspase-8 are essential for response to conatumumab.

\section{MATERIALS AND METHODS}

Cell lines and reagents. All human EWS cell lines and the breast carcinoma cell line MDA-MB-231 (MB-231) were maintained in RPMI-1640 with $10 \%$ fetal bovine serum and antibiotics. Human skeletal muscle-derived cells (SkMDC) were obtained from Cook Myosite (Pittsburgh, PA, USA) and cultured as per manufacturer's manual. Mouse antibodies against human DR4 (MAB347) and DR5 (MAB631) for cell-based assays were purchased from R\&D Systems (Minneapolis, MN, USA). The human DR5 antibody conatumumab was kindly provided by Amgen Inc. (Thousand Oaks, CA, USA). The goat anti-Fc antibody was obtained from Jackson Lab (Bar Harbor, ME, USA). The expression plasmids with CASP8 and enzymatic defective CASP ${ }^{\mathrm{mt}}$ (C360S) were provided by Drs Michael Lenardo and Lixin Zheng at the US National
Institutes of Health. DNA transfection was performed with lipofectamine as per manufacturer's manual (Invitrogen, Carlsbad, CA, USA).

Cell viability. Cell proliferation assays were performed with ATPLite reagent as previously described (Cao et al, 2008). All experiments were performed in triplicate and at least three repetitive experiments were performed for each result.

Immunoblots analysis. Cell and tissue lysates were prepared as previously described (Cao et al, 2008). The antibodies against DR4 and DR5 were from Imgenex (San Diego, CA, USA), FADD was obtained from BD Pharmingen (San Diego, CA, USA), FLIP, PARP, caspase- 3 , caspase- 8 , caspase-10, $\alpha$-tubulin, GAPDH, and actin were obtained from Cell Signaling Technology (Danvers, MA, USA).

Animal studies. Animal protocols were approved by Animal Care and Use Committee at NCI (Protocol GB-010) and the Johns Hopkins University Animal Care and Use Committee (Protocol MO13M231). Female 4- to 6-week-old CB17.B6-Prkdc scid mice were purchased from Charles River Laboratories (Wilmington, MA, USA). The animal study of Rh1 cells was performed with an intramuscular implantation model as previously described (Cao et al, 2008). Conatumumab treatment was initiated as described in the results. Conatumumab or a placebo antibody was given at $10 \mathrm{mg} \mathrm{kg}^{-1}$ i.p. once weekly, and tumour dimensions were measured using calipers.

For the mouse models using two histologically intact patientderived xenografts (PDX), EWS1 and EWS4, that express the EWS/FLI-1 fusion gene and have been multiply serially passaged (kind gifts from Dr. Chand Khanna, National Cancer Institute, Bethesda, MD, USA), tumour was first grown in a single immunedeficient NOD/SCID/IL-2R-null (NSG) mouse. When the tumour reached sufficient size, the mouse was killed and the tumour divided into fragments of $\sim 2 \mathrm{~mm}$ diameter. Intact fragments were washed in matrigel basement membrane matrix (BD Biosciences, San Jose, CA, USA) and subsequently surgically implanted subcutaneously in the flanks of NSG mice. Mice were randomised into control and treatment cohorts (five mice each) when the tumour was clearly palpable, typically at $5 \mathrm{~mm}$ in diameter. Conatumumab $\left(200 \mu \mathrm{g}\right.$ at $\left.2 \mathrm{mg} \mathrm{ml}^{-1}\right)$ or vehicle $(100 \mu \mathrm{l}$ sterile phosphate-buffered saline) was injected i.p. once weekly beginning immediately after palpable tumour was confirmed, and tumour dimensions were measured weekly using calipers, with volume calculated using an elliptical formula. Mice were killed at the time point when tumours were first measured to approach $20 \mathrm{~mm}$ in greatest diameter. For the growth and survival curves in Figure 5, end points were either killing or administrative censor at mean tumour diameter of greater than $15 \mathrm{~mm}$, whichever came first.

Statistical analysis. Statistical analyses were performed with Prism (GraphPad, La Jolla, CA, USA). All cell-based studies were done in triplicate. Data are presented as mean \pm s.e.m. Statistical comparisons were conducted using a two-way ANOVA test using Prism. Kaplan-Meier survival curves were compared using the Log-Rank Test. Statistical significance was defined as $P<0.05$.

\section{RESULTS}

The majority of the EWS cell lines are sensitive to a DR5 antibody. To determine the in vitro sensitivity of a panel of EWS cell lines to selective TRAIL receptor targeted agents, drug sensitivity analysis was performed using 12 EWS cell lines with various concentrations of TRAIL receptor DR4 or DR5-specific mouse monoclonal antibodies that were previously shown to induce death receptor-dependent cell death in the control breast cancer cell line MDA-MB-231 (Kang et al, 2011). The cells were 
treated with either DR4 or DR5 antibody at doses ranging between 1 and $1000 \mathrm{ng} \mathrm{ml}^{-1}$ for 3 days and cell viability was determined afterwards. Only two cell lines (RD-ES and TC71) were sensitive to both DR4 and DR5 antibodies (Figure 1A and B, Table 1). Eight of the remaining cell lines were selectively sensitive to the DR5 antibody, whereas two others (A4573 and LD) were resistant to both antibodies (Table 1). In contrast, primary human SkMDC were completely insensitive to treatment of DR4 or DR5 antibodies in vitro (Table 1). Immunoblot analysis showed that DR5 was expressed in all EWS cell lines; whereas DR4 was expressed at lower levels in most EWS cell lines, with the highest expression level in RD-ES, which is the most DR4 antibody-sensitive cell line among the tested EWS cell lines (Figure 1C, Table 1). Only one other EWS cell line, TC71, exhibited a modest sensitivity to DR4 antibody and a lower level of DR4 expression (Figure 1, Table 1). Thus, our results on death receptor antibody sensitivity profiling suggest that EWS cells are more susceptible to a DR5 agonist antibody, associated with higher levels of DR5 expression.

Human DR5 agonist antibody conatumumab is effective against EWS cells in vitro. Since the majority of EWS cell lines were sensitive to a DR5 antibody in vitro, we explored the therapeutic potential of an investigational agent, conatumumab, for EWS.
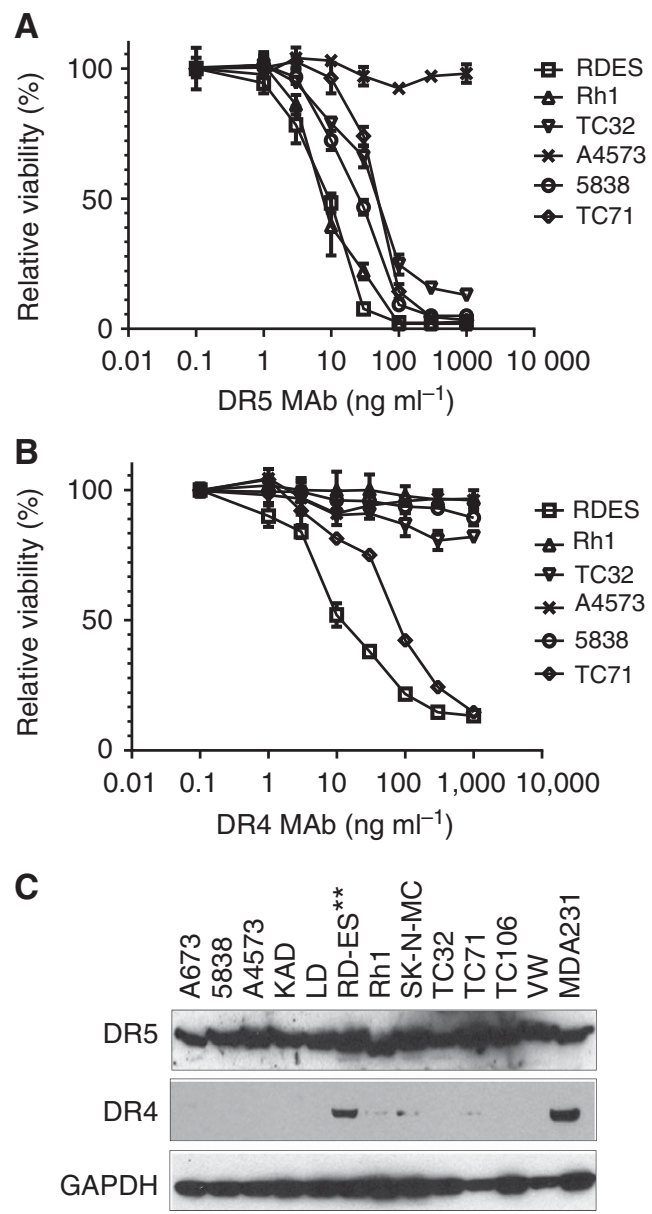

Figure 1. EWS cells are sensitive to DR5 agonist antibody induced cell death. (A) Cell viability analysis of representative EWS cell lines treated with mouse monoclonal DR5 antibody for $72 \mathrm{~h}$, followed by ATPLite assay. (B) Sensitivity of the EWS cell lines to mouse monoclonal antibodies against DR4. The cells were treated with indicated concentrations of the antibody for $72 \mathrm{~h}$ before the viability assay. (C) The expression of DR4 and DR5 in a panel of EWS cell lines was determined via immunoblots.
Conatumumab is a fully human agonist antibody to death receptor DR5 that has been shown to have both in vitro and in vivo activities against a number of selected solid tumour cell lines (Kaplan-Lefko et al, 2010). We examined the activity of this antibody against the same panel of EWS cell lines tested for mouse DR5 antibody. Conatumumab was previously reported to require crosslinking with protein $\mathrm{G}$ to be active (Kaplan-Lefko et al, 2010). We found that this antibody was only active in the presence of anti-Fc antibody (Figure 2A and B), in agreement with the previous findings. Nine of the EWS cell lines were very sensitive to conatumumab (Table 1). Only A4573 was completely resistant to conatumumab, whereas cell lines LD and TC106 showed a modest degree of reduced sensitivity (Table 1). Conatumumab treatment resulted in a rapid induction of apoptosis, as indicated by fragmented nuclei post treatment in the sensitive Rh1 cells, but not in the resistant A4573 cells (Figure 2C). Furthermore, clonogenic assay showed that the conatumumab treatment specifically prevented the formation of tumour cell colonies of the sensitive Rh1 cells (Figure 2D), but not the resistant A4573 cells. Thus, conatumumab appears to be an effective agent against the majority of the EWS cell lines in vitro and its activity requires antibody crosslinking.

Reduced caspase-8 expression is implicated in resistance to conatumumab in EWS. To better understand the cellular components important to the sensitivity of EWS cell lines to conatumumab, we analysed the levels of proteins present in the DISC, which is the key mediator of TRAIL-induced apoptosis. The expression of caspase- 8 is tightly correlated with EWS cellular sensitivity to conatumumab: it was absent in the most resistant A4573 cells, and expressed at substantially reduced levels in the partially resistant LD and TC106 cell lines (Figure 3A). It should be noted that a closely related gene, CASP10, is highly expressed in the most resistant cell line, A4573, suggesting that caspase-10 is insufficient to confer sensitivity to conatumumab in this cell line. No correlation between FADD, FLIP or caspase- 3 expression and sensitivity to conatumumab was seen. Further analysis showed that conatumumab induced caspase- 8 cleavage at $60 \mathrm{~min}$, associated with cleaved caspase- 3 and PARP, which are key markers of apoptosis (Figure 3B). In contrast, caspase-10 cleavage was not detected in the sensitive Rh1 cells (Figure 3B). In addition, conatumumab induces the caspase- 3 and PARP cleavage in KAD cells that do not express caspase-10. Further, conatumumab treatment did not lead to cleaved caspase- 3 and PARP in the resistant A4573 cells, which express caspase-10 but not caspase- 8 . Thus, caspase- 8 appears to be a key mediator for conatumumabinduced apoptosis in EWS cells.

To further prove that insufficient expression of caspase- 8 is the key determinant of resistance to conatumumab, we investigated whether the introduction of active caspase- 8 is sufficient to convert a resistant EWS cell line into a sensitive one. For the gene transfer experiment, we used a wild-type CASP8-GFP (green fluorescence protein) and a protease-defective $C A S P 8^{\mathrm{mt}}$-GFP with a C360S mutation at the active-site cysteine of the caspase domain (Siegel et al, 1998). Both the caspase-8-GFP and the protease-defective caspase- $8^{\mathrm{mt}}$-GFP could be expressed and detected in the resistant A4573 cells via transient gene transfection (Figure 3C). Following conatumumab + anti-Fc treatment, only the A4573 cells transfected with wild-type $C A S P 8$, but not with $C A S P 8^{\mathrm{mt}}$, exhibited significant reduction in cell viability (Figure $3 \mathrm{D}$ ). Thus, our results indicate that the absence of caspase- 8 expression is associated with resistance to conatumumab and that exogenous expression of CASP8 is sufficient to mediate conatumumab-induced apoptosis in resistant EWS cells, which is dependent on its catalytic activity.

In vivo activity of conatumumab against EWS xenografts. The anti-tumour activity of conatumumab was evaluated in two xenograft mouse models: a conatumumab-sensitive EWS cell line 
Table 1. Drug sensitivity of Ewing's sarcoma cell lines to mouse anti-DR4 and DR5, Conatumumab (CONA), and CONA + anti-Fc

\begin{tabular}{|c|c|c|c|c|c|c|c|c|}
\hline \multirow[b]{2}{*}{ Agent } & \multicolumn{2}{|c|}{ DR4 mouse Mab } & \multicolumn{2}{|c|}{ DR5 mouse Mab } & \multicolumn{2}{|c|}{ CONA } & \multicolumn{2}{|c|}{ CONA + Anti-Fc } \\
\hline & $\begin{array}{c}\text { IC50 } \\
\left(\mathrm{ng} \mathrm{ml}^{-1}\right)\end{array}$ & $\begin{array}{c}\text { Viability (\%) at } \\
1 \mu \mathrm{g} \mathrm{ml}^{-1}\end{array}$ & $\begin{array}{c}\text { IC50 } \\
\left(\mathrm{ng} \mathrm{ml}^{-1}\right)\end{array}$ & $\begin{array}{c}\text { Viability (\%) at } \\
1 \mu \mathrm{g} \mathrm{ml}^{-1}\end{array}$ & $\begin{array}{c}\text { IC50 } \\
\left(\mathrm{ng} \mathrm{ml}^{-1}\right)\end{array}$ & $\begin{array}{c}\text { Viability (\%) at } \\
1 \mu \mathrm{g} \mathrm{ml}^{-1}\end{array}$ & $\begin{array}{c}\text { IC50 } \\
\left(\mathrm{ng} \mathrm{ml}^{-1}\right)\end{array}$ & $\begin{array}{c}\text { Viability (\%) at } \\
1 \mu \mathrm{g} \mathrm{ml}^{-1}\end{array}$ \\
\hline $\mathrm{RH} 1$ & $>1000$ & 99.6 & 9.3 & 2.1 & $>1000$ & 81.1 & 2.3 & 5.1 \\
\hline TC71 & 88.1 & 25.1 & 47.5 & 3.5 & $>1000$ & 90.2 & 5.1 & 7.9 \\
\hline RD-ES & 13.2 & 15.5 & 11.1 & 3.0 & $>1000$ & 91.2 & 1.8 & 4.0 \\
\hline SKN-MC & $>1000$ & 102.0 & 28.9 & 10.6 & $>1000$ & 93.1 & 2.5 & 8.4 \\
\hline TC106 & $>1000$ & 66.1 & 10.1 & 33.1 & $>1000$ & 91.6 & 30.0 & 42.2 \\
\hline TC32 & $>1000$ & 82.7 & 12.6 & 33.4 & $>1000$ & 100.0 & 3.8 & 9.8 \\
\hline KAD & $>1000$ & 97.7 & 98.9 & 18.9 & $>1000$ & 94.9 & 3.9 & 15.9 \\
\hline A4573 & $>1000$ & 97.1 & $>1000$ & 99.9 & $>1000$ & 95.3 & $>1000$ & 92.3 \\
\hline A673 & $>1000$ & 54.0 & 9.2 & 7.2 & $>1000$ & 70.0 & 3.1 & 7.2 \\
\hline VW & $>1000$ & 88.3 & 7.0 & 18.4 & $>1000$ & 68.3 & 3,3 & 18.1 \\
\hline LD & $>1000$ & 92.1 & $>1000$ & 78.1 & $>1000$ & 96.7 & 700.9 & 42.8 \\
\hline 5838 & $>1000$ & 90.1 & 29.2 & 5.2 & $>1000$ & 100.0 & 3.9 & 7.9 \\
\hline MDA231 & 27.7 & 44.6 & 30.9 & 25.6 & $>1000$ & 95.9 & 3.0 & 11.6 \\
\hline SkMDC & $>1000$ & 100 & $>1000$ & 97 & $>1000$ & 100 & $>1000$ & 95 \\
\hline
\end{tabular}
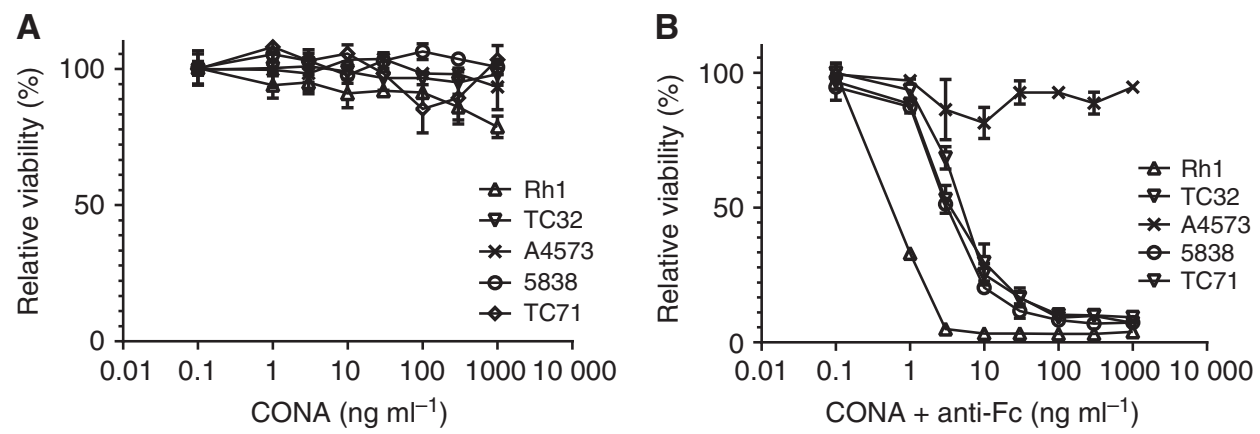

C

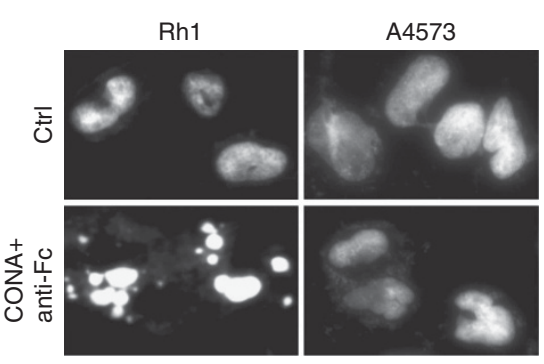

D
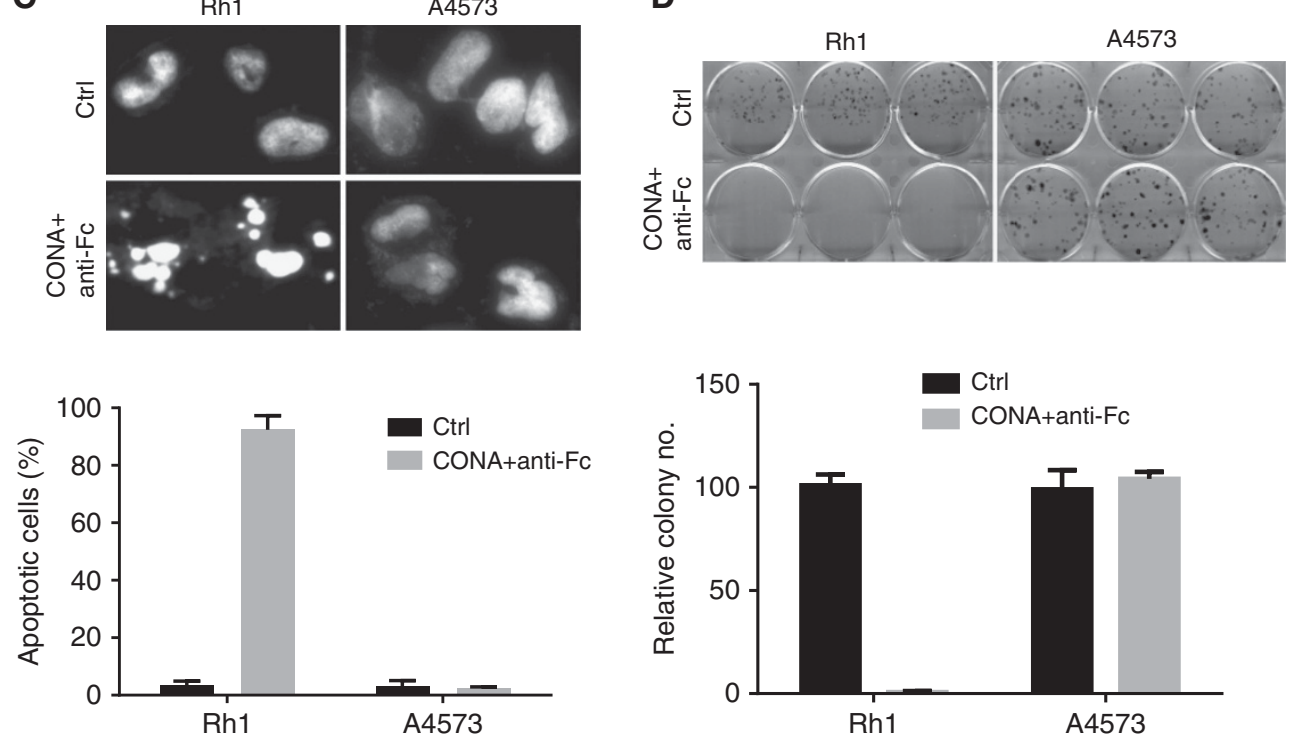

Figure 2. Conatumumab (CONA) crosslinked with anti-Fc antibody induces apoptosis in the sensitive EWS cells. (A) EWS cell lines treated with human DR5 antibody CONA alone for $72 \mathrm{~h}$, followed by viability assay. (B) EWS cell lines treated with CONA plus $1: 1$ ratio of goat anti-Fc antibody at the indicated doses for $72 \mathrm{~h}$ before the viability assay. (C) Both sensitive Rh1 and resistant A4573 cells were treated with vehicle or $1 \mu \mathrm{g} \mathrm{ml}^{-1}$ CONA + anti-Fc for $1 \mathrm{~h}$, fixed and stained with DAPI, and analysed for fragmented nuclei as a marker for apoptotic cells. The fractions of cells with apoptotic nuclei were counted and plotted. (D) Rh1 and A4573 cells were treated with vehicle or $1 \mu \mathrm{g} \mathrm{ml}{ }^{-1} \mathrm{CONA}+$ anti-Fc for 3 days, followed by continuous growth for 2 weeks. Colonies were counted and plotted. 
A

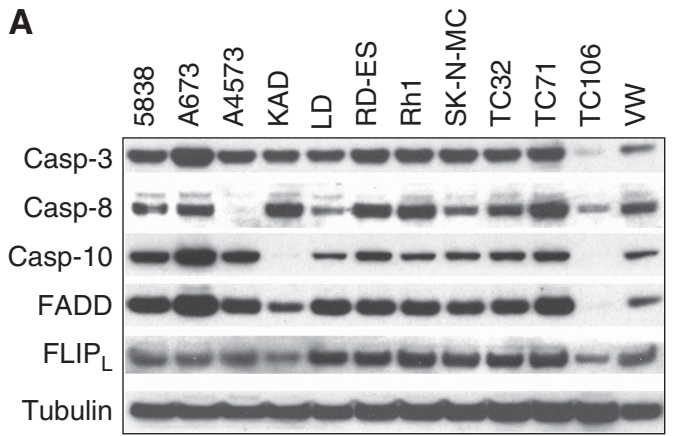

B

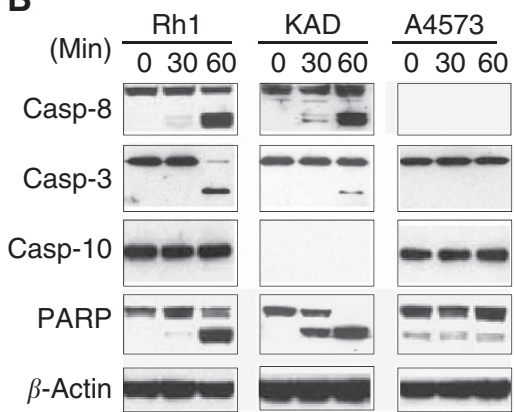

C

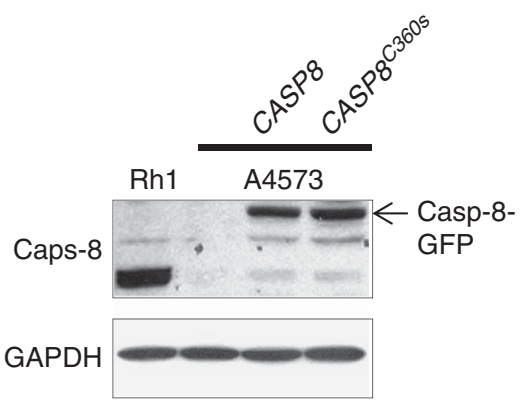

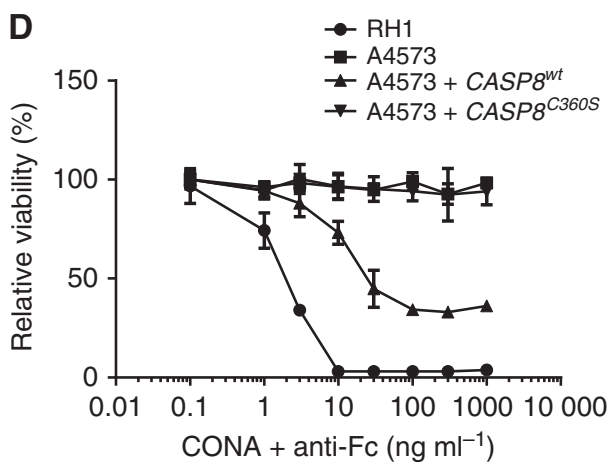

Figure 3. Association between caspase-8 expression and conatumumab sensitivity in EWS. (A) Analysis of EWS cell lines for the expression of proteins relevant to DISC and its associated initiator caspases 8 and 10. (B) Sensitive Rh1 and KAD, as well as resistant A4573, cells were treated with conatumumab + anti-Fc for the indicated time. The cell lysates were analysed for the cleavage of caspase-8, caspase-3, caspase-10, and PARP by immunoblots. (C) Ectopic expression of caspase-8-GFP. Conatumumab-resistant A4573 cells were transiently transfected with CASP8 or caspase-defective CASP8 ${ }^{\mathrm{mt}}$ (C360S) expression plasmids for 2 days. Cell lysates were analysed for caspase-8-GFP expression with immunoblot, using the sensitive Rh1 cells as a reference for caspase-8. (D) Ectopic expression of caspase-8 sensitises A4573 cells to CONA+anti-Fc induced cell death. After transient transfection with CASP8 or caspase-defective CASP8 ${ }^{\text {mt }}$ (C360S) expression plasmids for 2 days, the cells were then treated with CONA + anti-Fc for 3 days and analysed for cell viability. The sensitive Rh1 cells were used as a reference.

and a pair of EWS PDXs. The conatumumab-sensitive Rh1 cells were inoculated intramuscularly. After 4 weeks, the mice were randomised and treated with placebo or conatumumab, once weekly, for a total of up to 12 weeks. Tumour development was detected in mice from the control arm (5 out of 9) but not from the conatumumab-treated arm (0 out of 10$)$, a statistically significant difference $(P=0.0325)$ (Figure 4A). Kaplan-Meier survival analysis showed the tumour-free survival of the mice in the conatumumab-treated arm significantly better than those in the control arm (Log-Rank $P=0.0273$; Figure 4B). Thus, conatumumab has significant anti-tumour activity against the xenografts of Rh1cells.

We next examined the in vivo activity of conatumumab with a pair of EWS PDXs positive for the EWS/FLI-1 fusion gene. Mice implanted with either EWS1 or EWS4 PDX were randomised upon confirmation of palpable tumour and treated weekly with conatumumab or vehicle. In comparison with the vehicle control arm, conatumumab treatment had moderate, statistically significant activity in inhibiting the growth of the EWS4 PDX $(P=0.002)$, whereas it had no effect against EWS1 tumours $(P=0.27)$ (Figure 5A). Mice were followed until the control cohort required euthanasia due to tumour size. Conatumumab treatment prolonged the survival of mice implanted with the EWS4 (Log-Rank $P=0.011$ ), but not with the EWS1 PDX (Log-Rank $P=0.795$ ) (Figure 5B). Immunoblot analysis of tumour fragments from these mice showed that while both xenografts expressed comparable levels of DR5, EWS4 had a higher level of caspase-8 (Figure 5C). Treatment with conatumumab resulted in the induction of cleaved caspase-8 and PARP specifically in EWS4 tumours. Thus, we observed anti-tumour activity of conatumumab using EWS PDXs and found that the expression of caspase- 8 is associated with this activity.

\section{DISCUSSION}

To better manage metastatic and recurrent EWS, development of novel targeted therapies is required. Our finding that most cell lines are sensitive to a mouse monoclonal antibody against DR5 though not to a similar antibody to DR4 demonstrates that death receptor DR5 may be a rational target for EWS. An investigational human antibody to DR5, conatumumab, is highly toxic to most EWS cell lines in vitro in the presence of crosslinking anti-Fc antibody. Investigation into the mechanism of action reveals that conatumumab induces a rapid onset of apoptosis and one of the initiator protein caspase, caspase-8, but not caspase-10, is essential for the process. Using an in vivo xenograft mouse model with a sensitive EWS cell line, the administration of conatumumab exhibited moderate, statistically significant activity against tumour development and enhances mouse survival in comparison with the control group. By deploying a mouse PDX model, we show that conatumumab has some activity in inhibiting PDX tumour growth and prolonging survival against one PDX associated with higher expression of caspase- 8 .

Our work showed that caspase- 8 is the critically important initiator caspase in mediating DR5 signaling. The expression of caspase- 8 is highly correlative to conatumumab sensitive in EWS cell lines. The most resistant cell line, A4573, expresses little caspase-8. Despite an abundance of caspase-10, conatumumab cannot induce the activation of caspase signaling and PARP cleavage in A4573. In contrast, the conatumumab-sensitive cell line $\mathrm{KAD}$, which expresses caspase- 8 but completely lacks caspase-10, is competent in DR5 antibody induced cleavage of Caspase 3 and PARP. Importantly, transient transfection experiments show that 

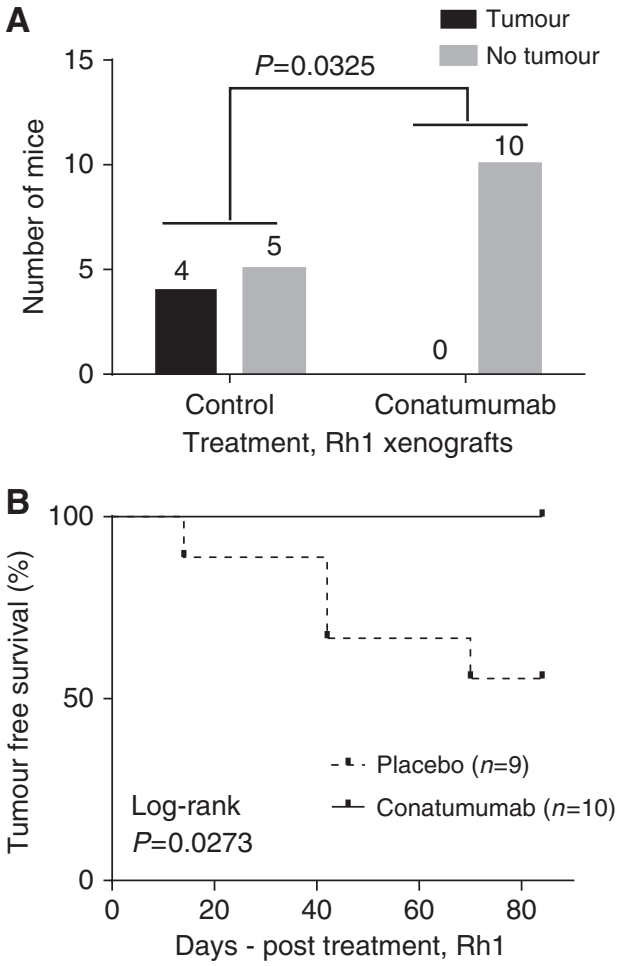

Figure 4. Anti-tumour activity of conatumumab in vivo using the sensitive Rh1 EWS cells. (A) Rh1, a sensitive EWS cell line, was inoculated intramuscularly into SCID mice. After 4 weeks, mice were randomised treated with conatumumab or placebo weekly for a total of 12 weeks. Tumour incidence was recorded and shown for both control and conatumumab arms. P-value was determined with Fisher's exact test. (B) Kaplan-Meier survival analysis of the mice with Rh1 xenograft to determine Log-Rank P-value of conatumumab treatment and control. over expression of wild-type, but not mutant, caspase- 8 restores the cellular sensitivity to conatumumab in a caspase-8-deficient cell line, further suggesting that caspase- 8 is the key limiting factor. This finding is in agreement with a previous publication in which caspase- 8 expression was suggested to be a key determinant for the sensitivity towards receptor-mediated apoptosis of a range of tumour cell lines, including EWS (Fulda et al, 2001). The observation that caspase- 8 expression could be restored with a demethylation agent (Fulda et al, 2001) would suggest the potential synergy between conatumumab and chromatin modifiers for enhanced activity.

Our previous study with another DR5 agonist antibody, drozitumab, demonstrated a dramatic effect in inducing the regression of established rhabdomyosarcoma sarcoma xenografts in vivo (Kang et al, 2011). In comparison, conatumumab treatment had more modest effects on the EWS4 PDX. One possibility is that the EWS4 PDX could be more heterogeneous than an established cell line and thus less susceptible to an agent. Another possibility may be due to the differences in the agents. The in vivo anti-tumour activity of drozitumab has been attributed to its crosslinking by $\mathrm{Fc} \gamma$ receptors ( $\mathrm{Fc} \gamma \mathrm{Rs})$ on immune cells that mediated the killing of xenograft tumour cells (Wilson et al, 2011). In contrast, it was recently reported that $\mathrm{F} c \gamma \mathrm{R}$ expressing immune cells were unable to enhance the ability of conatumumab to induce apoptosis of ovarian cancer cells in vitro (Tuthill et al, 2014). These findings suggest that the differences between drozitumab and conatumumab in tumour cell killing mediated by $\mathrm{Fc} \gamma \mathrm{R}$ of immune cells may account for a more moderate in vivo activity of conatumumab against the EWS PDX. Overall, our data indicate that EWS is a good target for a DR5 agonist antibody such as conatumumab, though some additional efforts in enhancing its in vivo therapeutic activity will be needed.

A previous clinical trial with the TRAIL ligand rhApo2L/TRAIL in patients with advanced cancers showed anti-tumour activity in two sarcoma patients, without evidence of activity in patients with carcinomas (Herbst et al, 2010a). Conatumumab has been safely

A

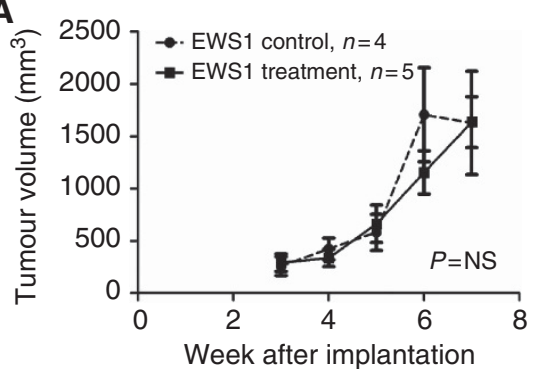

B

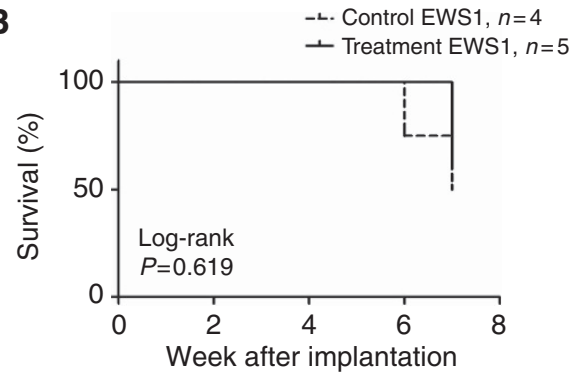

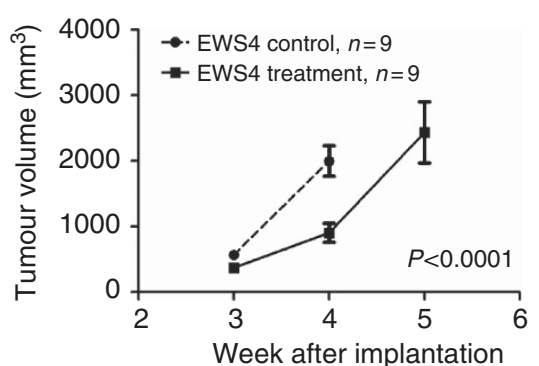

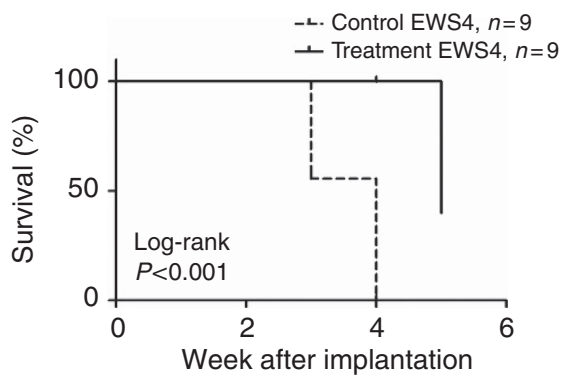

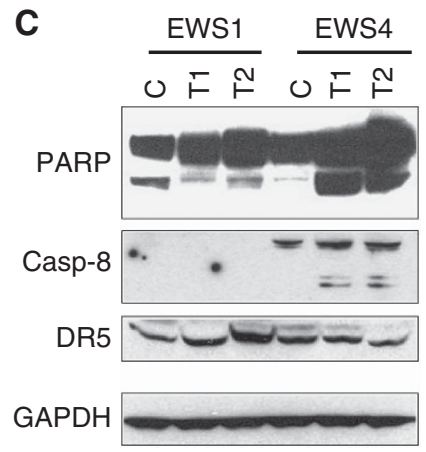

Figure 5. Anti-tumour activity of conatumumab in vivo using PDX xenograft tumours. (A) Two PDX xenograft tumours, EWS1 and EWS4, were surgically implanted subcutaneously in the flanks of NSG mice. Animals were randomised into control and treatment cohorts when the tumour reached around $5 \mathrm{~mm}$ in diameter. Once weekly, conatumumab or vehicle injection was performed and the tumour dimensions were measured. Animals were euthanised when the control cohort tumours reached $20 \mathrm{~mm}$ in maximal diameter. The tumour growth curves are shown. Comparison of the curves was performed using a two-way ANOVA test, and the $P$-value is shown. (B) Kaplan-Meier survival analysis of the same mice with EWS1 and EWS4 xenografts at the time the experiment was terminated. (C) EWS1 and EWS4 xenograft tissue lysates were made and subjected to immunoblots for the detection of DR5, and caspase-8 and PARP cleavage. C, control; T1/2, conatumumab-treated tumours. 
administered to adult patients with solid tumours (Herbst et al, 2010b). The only prior clinical trial evaluating conatumumab against sarcoma reported no benefit of the drug in terms of disease control (Demetri et al, 2012). In that study, doxorubicin with or without conatumumab was given to patients with a variety of subtypes of soft tissue sarcoma. The addition of DR5 antibody was found to be safe, but it did not increase response to doxorubicin. However, in the context of our findings, the conclusion that DR5 antibody is ineffective should be interpreted with caution. Conceivably, assessment of caspase- 8 expression as an individualised tumour marker, which was not performed, may have predicted response. We continue to believe that conatumumab holds promise as a targeted therapeutic therapy for appropriately selected patients, namely those with tumours expressing high levels of caspase-8. The combination with a DR5 ligand, such as rhApo2L/TRAIL, may also enhance its in vivo activity (Graves et al, 2014; Tuthill et al, 2014).

In conclusion, we report that EWS sensitivity to the activation of death receptor signaling pathway is primarily mediated through the DR5 receptor. DR4 receptor is expressed at a lower level and was previously shown to be absent at the surface of most EWS cell lines (Picarda et al, 2012). These data provide a rationale for the use of an anti-DR5 antibody such as conatumumab for the treatment of EWS. We have demonstrated that conatumumab is effective in vitro and in vivo against EWS and that tumour sensitivity is dependent on caspase- 8 expression. Thus, we believe our study provides the rationale for continued clinical evaluation of conatumumab against EWS. On the basis of these data, appropriate patient selection, specifically, the evaluation of caspase- 8 expression, will be of high importance to determine the clinical utility of conatumumab. This biomarker-driven rather than histologydriven approach comprises many of the facets of contemporary personalised cancer care and may be a model for testing of targeted therapies for other tumour types in the future.

\section{ACKNOWLEDGEMENTS}

We are very grateful to Dr Jeff Wiezorek of Amgen for providing conatumumab, Drs Michael Lenardo and Lixin Zheng for CASP8 and $C A S P 8^{\mathrm{mt}}$ (C360S) expression plasmids, Lee Helman for EWS cell lines, Chand Khanna for EWS PDX tumours, and Arnulfo Mendoza for excellent technical assistance. This research was supported by the Intramural Research Program of the US National Cancer Institute (NCI). This project was also funded in part with federal funds from the NCI, NIH, under contract HHSN261200800001E. The Giant Food Children's Cancer Research Fund also helped support this work in part. The content of this publication does not necessarily reflect the views or policies of the Department of Health and Human Services, nor does mention of trade names, commercial products, or organisations imply endorsement by the US Government. No potential financial or personal conflict of interest.

\section{CONFLICT OF INTEREST}

The authors declare no conflict of interest.

\section{REFERENCES}

Adams C, Totpal K, Lawrence D, Marsters S, Pitti R, Yee S, Ross S, Deforge L, Koeppen H, Sagolla M, Compaan D, Lowman H, Hymowitz S,

Ashkenazi A (2008) Structural and functional analysis of the interaction between the agonistic monoclonal antibody Apomab and the proapoptotic receptor DR5. Cell Death Differ 15(4): 751-761.
Ashkenazi A (2008) Directing cancer cells to self-destruct with pro-apoptotic receptor agonists. Nat Rev Drug Discov 7(12): 1001-1012.

Ashkenazi A, Herbst RS (2008) To kill a tumor cell: the potential of proapoptotic receptor agonists. J Clin Invest 118(6): 1979-1990.

Balamuth NJ, Womer RB (2010) Ewing's sarcoma. Lancet Oncol 11(2): 184-192.

Cao L, Yu Y, Darko I, Currier D, Mayeenuddin LH, Wan X, Khanna C, Helman LJ (2008) Addiction to elevated insulin-like growth factor I receptor and initial modulation of the AKT pathway define the responsiveness of rhabdomyosarcoma to the targeting antibody. Cancer Res 68(19): 8039-8048.

Cohn AL, Tabernero J, Maurel J, Nowara E, Sastre J, Chuah BY, Kopp MV, Sakaeva DD, Mitchell EP, Dubey S, Suzuki S, Hei YJ, Galimi F,

McCaffery I, Pan Y, Loberg R, Cottrell S, Choo SP (2013) A randomized, placebo-controlled phase 2 study of ganitumab or conatumumab in combination with FOLFIRI for second-line treatment of mutant KRAS metastatic colorectal cancer. Ann Oncol 24(7): 1777-1785.

Cotterill SJ, Ahrens S, Paulussen M, Jurgens HF, Voute PA, Gadner H, Craft AW (2000) Prognostic factors in Ewing's tumor of bone: analysis of 975 patients from the European Intergroup Cooperative Ewing's Sarcoma Study Group. J Clin Oncol 18(17): 3108-3114.

Delattre O, Zucman J, Plougastel B, Desmaze C, Melot T, Peter M, Kovar H, Joubert I, de Jong P, Rouleau G et al. (1992) Gene fusion with an ETS DNA-binding domain caused by chromosome translocation in human tumours. Nature 359(6391): 162-165.

Demetri GD, Le Cesne A, Chawla SP, Brodowicz T, Maki RG, Bach BA, Smethurst DP, Bray S, Hei YJ, Blay JY (2012) First-line treatment of metastatic or locally advanced unresectable soft tissue sarcomas with conatumumab in combination with doxorubicin or doxorubicin alone: a phase I/II open-label and double-blind study. Eur J Cancer 48(4): 547-563.

Fulda S, Kufer MU, Meyer E, van Valen F, Dockhorn-Dworniczak B, Debatin KM (2001) Sensitization for death receptor- or drug-induced apoptosis by re-expression of caspase- 8 through demethylation or gene transfer. Oncogene 20(41): 5865-5877.

Graves JD, Kordich JJ, Huang T-H, Piasecki J, Bush TL, Sullivan T, Foltz IN, Chang W, Douangpanya H, Dang T, O’Neill JW, Mallari R, Zhao X, Branstetter DG, Rossi JM, Long AM, Huang X, Holland PM (2014) Apo2L/TRAIL and the death receptor 5 agonist antibody AMG 655 cooperate to promote receptor clustering and antitumor activity. Cancer Cell 26(2): 177-189.

Herbst RS, Eckhardt SG, Kurzrock R, Ebbinghaus S, O’Dwyer PJ, Gordon MS, Novotny W, Goldwasser MA, Tohnya TM, Lum BL, Ashkenazi A, Jubb AM, Mendelson DS (2010a) Phase I dose-escalation study of recombinant human Apo2L/TRAIL, a dual proapoptotic receptor agonist, in patients with advanced cancer. J Clin Oncol 28(17): 2839-2846.

Herbst RS, Kurzrock R, Hong DS, Valdivieso M, Hsu CP, Goyal L, Juan G, Hwang YC, Wong S, Hill JS, Friberg G, LoRusso PM (2010b) A first-in-human study of conatumumab in adult patients with advanced solid tumors. Clin Cancer Res 16(23): 5883-5891.

Hughes MA, Harper N, Butterworth M, Cain K, Cohen GM, MacFarlane M (2009) Reconstitution of the death-inducing signaling complex reveals a substrate switch that determines CD95-mediated death or survival. Mol Cell 35(3): 265-279.

Jin H, Yang R, Ross J, Fong S, Carano R, Totpal K, Lawrence D, Zheng Z, Koeppen H, Stern H, Schwall R, Ashkenazi A (2008) Cooperation of the agonistic DR5 antibody apomab with chemotherapy to inhibit orthotopic lung tumor growth and improve survival. Clin Cancer Res 14(23): 7733-7740.

Jin Z, Li Y, Pitti R, Lawrence D, Pham VC, Lill JR, Ashkenazi A (2009) Cullin3-based polyubiquitination and p62-dependent aggregation of caspase-8 mediate extrinsic apoptosis signaling. Cell 137(4): 721-735.

Kang Z, Chen JJ, Yu Y, Li B, Sun SY, Zhang B, Cao L (2011) Drozitumab, a human antibody to death receptor 5 , has potent antitumor activity against rhabdomyosarcoma with the expression of caspase- 8 predictive of response. Clin Cancer Res 17(10): 3181-3192.

Kaplan-Lefko PJ, Graves JD, Zoog SJ, Pan Y, Wall J, Branstetter DG, Moriguchi J, Coxon A, Huard JN, Xu R, Peach ML, Juan G, Kaufman S, Chen Q, Bianchi A, Kordich JJ, Ma M, Foltz IN, Gliniak BC (2010) Conatumumab, a fully human agonist antibody to death receptor 5 , induces apoptosis via caspase activation in multiple tumor types. Cancer Biol Ther 9(8): 618-631. 
Kischkel FC, Lawrence DA, Chuntharapai A, Schow P, Kim KJ, Ashkenazi A (2000) Apo2L/TRAIL-dependent recruitment of endogenous FADD and caspase-8 to death receptors 4 and 5. Immunity 12(6): 611-620.

Kischkel FC, Lawrence DA, Tinel A, LeBlanc H, Virmani A, Schow P, Gazdar A, Blenis J, Arnott D, Ashkenazi A (2001) Death receptor recruitment of endogenous caspase-10 and apoptosis initiation in the absence of caspase-8. J Biol Chem 276(49): 46639-46646.

Locksley RM, Killeen N, Lenardo MJ (2001) The TNF and TNF receptor superfamilies: integrating mammalian biology. Cell 104(4): 487-501.

Luster TA, Carrell JA, McCormick K, Sun D, Humphreys R (2009) Mapatumumab and lexatumumab induce apoptosis in TRAIL-R1 and TRAIL-R2 antibody-resistant NSCLC cell lines when treated in combination with bortezomib. Mol Cancer Ther 8(2): 292-302.

Mitsiades N, Poulaki V, Mitsiades C, Tsokos M (2001) Ewing's sarcoma family tumors are sensitive to tumor necrosis factor-related apoptosisinducing ligand and express death receptor 4 and death receptor 5. Cancer Res 61(6): 2704-2712.

Paz-Ares L, Balint B, de Boer RH, van Meerbeeck JP, Wierzbicki R, De Souza P, Galimi F, Haddad V, Sabin T, Hei YJ, Pan Y, Cottrell S, Hsu CP, RamLau R (2013) A randomized phase 2 study of paclitaxel and carboplatin with or without conatumumab for first-line treatment of advanced non-small-cell lung cancer. J Thorac Oncol 8(3): 329-337.

Picarda G, Lamoureux F, Geffroy L, Delepine P, Montier T, Laud K, Tirode F, Delattre O, Heymann D, Redini F (2010) Preclinical evidence that use of TRAIL in Ewing's sarcoma and osteosarcoma therapy inhibits tumor growth, prevents osteolysis, and increases animal survival. Clin Cancer Res 16(8): 2363-2374.

Picarda G, Surget S, Guiho R, Teletchea S, Berreur M, Tirode F, Pellat-Deceunynck C, Heymann D, Trichet V, Redini F (2012) A functional, new short isoform of death receptor 4 in Ewing's sarcoma cell lines may be involved in TRAIL sensitivity/resistance mechanisms. Mol Cancer Res 10(3): 336-346.

Safa AR, Day TW, Wu CH (2008) Cellular FLICE-like inhibitory protein (C-FLIP): a novel target for cancer therapy. Curr Cancer Drug Targets 8(1): $37-46$
Siegel RM, Martin DA, Zheng L, Ng SY, Bertin J, Cohen J, Lenardo MJ (1998) Death-effector filaments: novel cytoplasmic structures that recruit caspases and trigger apoptosis. J Cell Biol 141(5): 1243-1253.

Tirode F, Laud-Duval K, Prieur A, Delorme B, Charbord P, Delattre O (2007) Mesenchymal stem cell features of Ewing tumors. Cancer Cell 11(5): 421-429.

Tuthill MH, Montinaro A, Zinngrebe J, Prieske K, Draber P, Prieske S, Newsom-Davis T, von Karstedt S, Graves J, Walczak H (2014) TRAIL-R2-specific antibodies and recombinant TRAIL can synergise to kill cancer cells. Oncogene 34(16): 2138-2144.

Van Valen F, Fulda S, Truckenbrod B, Eckervogt V, Sonnemann J, Hillmann A, Rodl R, Hoffmann C, Winkelmann W, Schafer L, Dockhorn-Dworniczak B, Wessel T, Boos J, Debatin KM, Jurgens H (2000) Apoptotic responsiveness of the Ewing's sarcoma family of tumours to tumour necrosis factor-related apoptosis-inducing ligand (TRAIL). Int J Cancer 88(2): 252-259.

Wagner KW, Punnoose EA, Januario T, Lawrence DA, Pitti RM, Lancaster K, Lee D, von Goetz M, Yee SF, Totpal K, Huw L, Katta V, Cavet G, Hymowitz SG, Amler L, Ashkenazi A (2007) Death-receptor O-glycosylation controls tumor-cell sensitivity to the proapoptotic ligand Apo2L/TRAIL. Nat Med 13(9): 1070-1077.

Wilson NS, Yang B, Yang A, Loeser S, Marsters S, Lawrence D, Li Y, Pitti R, Totpal K, Yee S, Ross S, Vernes JM, Lu Y, Adams C, Offringa R, Kelley B, Hymowitz S, Daniel D, Meng G, Ashkenazi A (2011) An Fcgamma receptor-dependent mechanism drives antibody-mediated target-receptor signaling in cancer cells. Cancer Cell 19(1): 101-113.

Zhang Y, Zhang B (2008) TRAIL resistance of breast cancer cells is associated with constitutive endocytosis of death receptors 4 and 5. Mol Cancer Res 6(12): 1861-1871.

This work is published under the standard license to publish agreement. After 12 months the work will become freely available and the license terms will switch to a Creative Commons AttributionNonCommercial-Share Alike 4.0 Unported License. 